Shuai Zhou, Zhisu Liu, and Jianjun Zhang*

\title{
Groundstates for Choquard type equations with weighted potentials and Hardy-Littlewood-Sobolev lower critical exponent
}

https://doi.org/10.1515/anona-2020-0186

Received March 24, 2021; accepted April 13, 2021.

Abstract: We are concerned with a class of Choquard type equations with weighted potentials and HardyLittlewood-Sobolev lower critical exponent

$$
-\Delta u+V(x) u=\left(I_{\alpha} \star\left[Q(x)|u|^{\frac{N+\alpha}{N}}\right]\right) Q(x)|u|^{\frac{\alpha}{N}-1} u, \quad x \in \mathbb{R}^{N} .
$$

By using variational approaches, we investigate the existence of groundstates relying on the asymptotic behaviour of weighted potentials at infinity. Moreover, non-existence of non-trivial solutions is also considered. In particular, we give a partial answer to some open questions raised in [D. Cassani, J. Van Schaftingen and J. J. Zhang, Groundstates for Choquard type equations with Hardy-Littlewood-Sobolev lower critical exponent, Proceedings of the Royal Society of Edinburgh, Section A Mathematics, 150(2020), 1377-1400].

Keywords: Ground states, Choquard equation, Hardy-Littlewood-Sobolev inequality, critical growth

MSC: 35B33, 35J61

\section{Introduction and main results}

In this paper, we are concerned with the following class of nonlocal equations

$$
-\Delta u+V(x) u=\left(I_{\alpha}{ }^{\star} F(x, u)\right) f(x, u), \quad x \in \mathbb{R}^{N},
$$

where $N \geq 3, V \in C\left(\mathbb{R}^{N}, \mathbb{R}\right)$ and $I_{\alpha}$ is the Riesz potential given for each $x \in \mathbb{R}^{N} \backslash\{0\}$ by

$$
I_{\alpha}(x):=\frac{A_{\alpha}}{|x|^{N-\alpha}}, \quad \text { where } \quad A_{\alpha}=\frac{\Gamma((N-\alpha) / 2)}{\Gamma(\alpha / 2) \pi^{N / 2} 2^{\alpha}} \quad \text { and } \alpha \in(0, N) .
$$

Here $\Gamma$ is the Euler gamma function and $F$ is the primitive function of $f \in C\left(\mathbb{R}^{N} \times \mathbb{R}, \mathbb{R}\right)$ with respect to $u$ and satisfies $F(0)=0$. In the literature, problem (1.1) is known as Choquard's type equation. Set $F(x, u)=|u|^{p} / p$ and $V \equiv a$, problem (1.1) becomes

$$
-\Delta u+a u=\left(I_{\alpha} \star|u|^{p}\right)|u|^{p-2} u, x \in \mathbb{R}^{N} .
$$

Shuai Zhou, College of Mathematics and Statistics, Chongqing Jiaotong University, Chongqing 400074, PR China, E-mail: 916164424@qq.com

Zhisu Liu, Center for Mathematical Sciences, China University of Geosciences, Wuhan, Hubei, 430074, PR China, E-mail: liuzhisu@cug.edu.cn

*Corresponding Author: Jianjun Zhang, College of Mathematics and Statistics, Chongqing Jiaotong University, Chongqing 400074, PR China, E-mail: zhangjianjun09@tsinghua.org.cn 
For $N=3, \alpha=2$ and $p=2,(1.2)$ reduces to

$$
-\Delta u+a u=\left(I_{2}{ }^{2} u^{2}\right) u, x \in \mathbb{R}^{3} .
$$

It seems that such equations appear first in the seminal work of S.I. Pekar '54 [23], modeling the quantum Polaron and later were introduced by Choquard to study steady states of the one component plasma approximation in the context of Hartree-Fock theory [11]. Problem (1.1) has a variational structure, in the sense that $H^{1}$-solutions to (1.1) turn out to be critical points of the energy functional

$$
E(u)=\frac{1}{2} \int_{\mathbb{R}^{N}}\left(|\nabla u|^{2}+V(x) u^{2}\right) \mathrm{d} x-\int_{\mathbb{R}^{N}}\left[I_{\alpha} \star F(x, u)\right] F(x, u) \mathrm{d} x .
$$

Due to the presence of convolution, problem (1.1) is nonlocal. In contrast with local problems, Choquard type equations carry some extra difficulty due to the nonlocal nature. By using a rearrangement approach, E. Lieb in [12] proved existence and uniqueness of positive solutions to (1.3). Subsequently, multiplicity results for (1.3) were obtained by P.L. Lions $[14,15]$ via the variational methods. Initiated by the papers of E. Lieb [12] and P.L. Lions $[14,15]$, Choquard equations have attracted a considerable attention in the past decades. We refer to [21] for a survey.

In [19], V. Moroz and J. Van Schaftingen established existence of ground state solutions to (1.2). Thanks to a Pohozǎev identity, they show that (1.2) admits a nontrivial solution in $H^{1}\left(\mathbb{R}^{N}\right)$ if and only if

$$
\frac{N+\alpha}{N}<p<\frac{N+\alpha}{N-2} .
$$

The endpoints $2_{\alpha, \star}:=\frac{N+\alpha}{N}$ and $2_{\alpha}^{\star}:=\frac{N+\alpha}{N-2}$ are sometimes called lower and upper Hardy-Littlewood-Sobolev critical exponents respectively in the sense of the Hardy-Littlewood-Sobolev inequality (see Lemma 2.1 below). Later, V. Moroz and J. Van Schaftingen [22] considered the autonomous form of Choquard equation (1.1)

$$
-\Delta u+u=\left(I_{\alpha} \star F(u)\right) f(u), \quad x \in \mathbb{R}^{N}
$$

and investigated existence, symmetry and regularity of groundstates to problem (1.5) under almost necessary conditions on the nonlinearity $F$ in the spirit of Berestycki and Lions. For the upper critical case, F. Gao and $M$. Yang [9] establish existence and nonexistence of solutions to the following Brezis-Nirenberg type problem of Choquard equation in bounded domains $\Omega \subset \mathbb{R}^{N}(N \geq 3)$

$$
-\Delta u=\left(\int_{\Omega} \frac{|u(y)|^{2_{\alpha}^{*}}}{|x-y|^{N-\alpha}} \mathrm{d} y\right)|u|^{2_{\alpha}^{*}-2} u+\lambda u, u \in H_{0}^{1}(\Omega) .
$$

By using the penalization argument introduced by J. Byeon and L. Jeanjean [4], D. Cassani and J. Zhang [6] investigated singularly perturbed problems related to equation (1.5) involving upper critical exponent

$$
-\varepsilon^{2} \Delta u+V(x) u=\varepsilon^{-\alpha}\left(I_{\alpha}{ }^{\star} F(u)\right) f(u), \quad x \in \mathbb{R}^{N}
$$

and obtained existence of single peak solutions around local minimal points of the potential $V$. With the help of the concentration compactness principle in the Choquard-type setting, S. Liang, P. Pucci and B. Zhang [16] established multiplicity results for Choquard-Kirchhoff type equations with Hardy-Littlewood-Sobolev critical exponents

$$
-\left(a+b \int_{\mathbb{R}^{N}}|\nabla u|^{2} \mathrm{~d} x\right) \Delta u=\alpha k(x)|u|^{q-2} u+\beta\left(\int_{\mathbb{R}^{N}} \frac{|u(y)|^{2_{\alpha}^{*}}}{|x-y|^{N-\alpha}} \mathrm{d} y\right)|u|^{2_{\alpha}^{*}-2} u, x \in \mathbb{R}^{N}
$$

Compared with the upper critical case, the lower critical case has been less considered. Combining variational arguments with the concentration-compactness principle [13], V. Moroz and J. Van Schaftingen [20] considered Choquard equations with a purely lower critical nonlinearity

$$
-\Delta u+V(x) u=\left(I_{\alpha}{ }^{\star}|u|^{\frac{N+\alpha}{N}}\right)|u|^{\frac{\alpha}{N}-1} u, \quad x \in \mathbb{R}^{N}
$$


and established a sufficient condition on existence of groundstates to problem (1.6). Subsequently, D. Cassani, J. Van Schaftingen and J. Zhang [7] investigated existence and nonexistence of groundstates to problem (1.6) and give a partial answer to some open questions raised in [7]. By variational methods, J. Van Schaftingen and J. Xia [25] proved existence of ground state solutions to Choquard equations with lower critical exponent and a subcritical perturbation. In [24], J. Seok considered problem (1.5) with both upper and lower critical exponents and obtained existence of nontrivial solutions in the higher dimensional case. For the related results on the Choquard equations with upper critical growth in the fractional setting and for the planar Choquard equations, we refer to $[1,2,5,8,17,26]$ and references therein.

We point out that due to the presence of the lower critical exponent $2_{\alpha, \star}$, the problem has a lack of compactness. Similarly to Sobolev critical problems, a Brezis-Nirenberg argument can be adopted to recover compactness. Actually, by imposing some suitable conditions on $N, \alpha$ and nonlinearities, one can get a candidate minimax value below a threshold, under which the compactness condition holds. In [7], compared with the high dimensional case $N \geq 3$, dimension $N=3$ becomes more tough. Precisely, in [7] to recover compactness in the three dimensional case, one sufficient condition is established on $\alpha$ as follows:

$$
\frac{3}{2}<\alpha<3
$$

Moreover, a natural question is whether such restriction is necessary or not for the existence of groundstates.

In this paper, we consider the following class of equations

$$
-\Delta u+V(x) u=\left(I_{\alpha}{ }^{\star}\left[Q(x)|u|^{\frac{N+\alpha}{N}}\right]\right) Q(x)|u|^{\frac{\alpha}{N}-1} u, \quad x \in \mathbb{R}^{N}
$$

and show that condition (1.7) can be replaced by demanding in the presence of weights a suitable asymptotic behavior at infinity. In the following, we perform the variational method to study existence and nonexistence of ground states to (1.8).

The associated functional with the Choquard equation (1.8) is given for any function $u: \mathbb{R}^{N} \rightarrow \mathbb{R}$ by

$$
\begin{aligned}
I(u)= & \frac{1}{2} \int_{\mathbb{R}^{N}}\left(|\nabla u|^{2}+V(x)|u|^{2}\right) \mathrm{d} x \\
& -\frac{N}{2(N+\alpha)} \int_{\mathbb{R}^{N}}\left(I_{\alpha} \star\left[Q(x)|u|^{\frac{N+\alpha}{N}}\right]\right) Q(x)|u|^{\frac{N+\alpha}{N}} \mathrm{~d} x
\end{aligned}
$$

We assume that $V$ and $Q$ satisfy

$\left(V_{1}\right) \inf _{x \in \mathbb{R}^{N}} V(x)>0, \lim _{|x| \rightarrow \infty} V(x)=1$;

$\left(V_{2}\right)$ There exists $\mu \in \mathbb{R}$ such that

$$
\lim _{|x| \rightarrow \infty}(1-V(x))|x|^{2}=\mu
$$

$\left(Q_{1}\right) \inf _{x \in \mathbb{R}^{N}} Q(x)>0, \lim _{|x| \rightarrow \infty} Q(x)=1$;

$\left(Q_{2}\right)$ There exist $\beta \geq 0$ and $v_{\beta} \in \mathbb{R}$ such that

$$
\lim _{|x| \rightarrow \infty}(Q(x)-1)|x|^{\beta}=v_{\beta} .
$$

Our main results are the following:

Theorem 1.1. Assume $\left(V_{1}\right),\left(V_{2}\right),\left(Q_{1}\right),\left(Q_{2}\right)$ hold, then (1.8) admits a positive ground state solution, provided one of the following conditions holds

(i) $\beta=0, \mu>\frac{N^{2}(N-2)}{4(N+1)}, \inf _{x \in \mathbb{R}^{N}} Q(x) \geq 1$;

(ii) $0<\beta<2, v_{\beta}>0$;

(iii) $\beta>2, \mu>\frac{N^{2}(N-2)}{4(N+1)}$. 
Combining a Pohozǎev identity(see Proposition 2.1 below) with Hardy's inequality, we have the following non-existence result for problem (1.8).

Theorem 1.2. Assume $V, Q \in C^{1}\left(\mathbb{R}^{N}\right) \cap L^{\infty}\left(\mathbb{R}^{N}\right)$ and

(VQ) $\left\{\begin{array}{l}\sup _{x \in \mathbb{R}^{N}}|x|^{2}\langle x, \nabla V(x)\rangle<\frac{(N-2)^{2}}{2}, \\ \inf _{x \in \mathbb{R}^{N}}\langle x, \nabla Q(x)\rangle \geq 0,\end{array}\right.$

then (1.8) admits in $H^{1}\left(\mathbb{R}^{N}\right) \cap W_{\text {loc }}^{2,2}\left(\mathbb{R}^{N}\right)$ only trivial solution.

Remark 1.1. The restriction $\inf _{x \in \mathbb{R}^{N}} V(x)>0$ is used to guarantee $c_{\star}$ (see below) is finite. It can be replaced by a weaker condition inf $\sigma(-\Delta+V)>0$.

Remark 1.2. In the case $\beta=2$, Theorem 1.1 is still valid if $\mu>\mu \star$, where

$$
\mu_{\star}=\frac{N^{2}(N-2)}{4(N+1)}-\frac{2 N v_{\beta}}{(N+\alpha) c_{\infty}}\left(\int_{\mathbb{R}^{N}} \frac{|U(x)|^{2}}{|x|^{2}} \mathrm{~d} x\right)^{-1} \int_{\mathbb{R}^{N}}\left(I_{\alpha} \star|U|^{\frac{N+\alpha}{N}}\right)\left[|x|^{-2}|U|^{\frac{N+\alpha}{N}}\right] \mathrm{d} x .
$$

Here $c_{\infty}$ and $U$ are given in Section 2.

As a special case, for the external Schrödinger potential $V_{\mu, \nu}: \mathbb{R}^{N} \rightarrow \mathbb{R}$

$$
V_{\mu, v}(x)=1-\frac{\mu}{v^{2}+|x|^{2}}, \quad \text { for } \mu \in \mathbb{R}, v>0 \text { and } x \in \mathbb{R}^{N}
$$

and the weighted potential $Q_{\beta}: \mathbb{R}^{N} \rightarrow \mathbb{R}$

$$
Q_{\beta}(x)=1+\frac{v_{\beta}}{1+|x|^{\beta}}, \quad \text { for } v_{\beta} \in \mathbb{R}, \beta \geq 0 \text { and } x \in \mathbb{R}^{N},
$$

problem (1.8) reduces to the following

$$
-\Delta u+V_{\mu, v}(x) u=\left(I_{\alpha} \star\left[Q_{\beta}(x)|u|^{\frac{N+\alpha}{N}}\right]\right) Q_{\beta}(x)|u|^{\frac{\alpha}{N}-1} u, \quad x \in \mathbb{R}^{N} .
$$

Denote by $\mu^{v}$ the best constant of the embedding $H^{1}\left(\mathbb{R}^{N}\right) \leftrightarrow L^{2}\left(\mathbb{R}^{N},\left(v^{2}+|x|^{2}\right)^{-1} \mathrm{~d} x\right)$, that is,

$$
\mu^{v}:=\inf _{u \in H^{1}\left(\mathbb{R}^{N}\right) \backslash\{0\}} \frac{\int_{\mathbb{R}^{N}}|\nabla u|^{2}+|u|^{2}}{\int_{\mathbb{R}^{N}} \frac{|u(x)|^{2}}{v^{2}+|x|^{2}} \mathrm{~d} x} .
$$

It has been shown in [7] that $\mu^{v}>\frac{(N-2)^{2}}{4}+v^{2}$.

Corollary 1.1. Problem (1.9) admits a positive ground state solution, provided one of the following conditions holds

(i) $\beta=0, \frac{N^{2}(N-2)}{4(N+1)}<\mu<\mu^{v}, v_{\beta}=0$;

(ii) $0<\beta<2, \mu<\mu^{v}, v_{\beta}>0$;

(iii) $\beta>2, \frac{N^{2}(N-2)}{4(N+1)}<\mu<\mu^{v}, v_{\beta}>-1$

and has no non-trivial solutions if $\mu<\frac{(N-2)^{2}}{4}$ and $v_{\beta} \leq 0$.

In the case $\mu>\mu^{v}$, the operator $-\Delta+V_{\mu, v}$ is not positively definite and the problem becomes more complicated. By the linking theorem, we have the following result. 
Theorem 1.3. Assume that either $N \geq 4$ or $N=3$, $\operatorname{ker}\left(-\Delta+V_{\mu, v}\right)=\{0\}$. If $\mu>\max \left\{\mu^{v}, \frac{N^{2}(N-2)}{4(N+1)}\right\}$, then (1.8) admits a ground state solution (necessarily sign changing) provided when $N=3$, one of the followings holds

(i) $\beta=0, v_{\beta}=0, \frac{3}{2}<\alpha<3$;

(ii) $0<\beta<\min \left\{\frac{9}{6-\alpha}, 2\right\}, v_{\beta}>0$

(iii) $\beta>2, v_{\beta}>-1, \frac{3}{2}<\alpha<3$;

and when $N \geq 4$, one of the followings holds

(iv) $\beta=0, v_{\beta}=0$;

(v) $0<\beta<2, v_{\beta}>0$;

(vi) $\beta>2, v_{\beta}>-1$.

\section{Notations.}

- $\|u\|_{p}:=\left(\int_{\mathbb{R}^{N}}|u|^{p} \mathrm{~d} x\right)^{1 / p}$ for $u \in L^{p}\left(\mathbb{R}^{N}\right), p \in[1, \infty)$.

- $\|u\|=\left(\|\nabla u\|_{2}^{2}+\|u\|_{2}^{2}\right)^{\frac{1}{2}}, u \in H^{1}\left(\mathbb{R}^{N}\right)$.

\section{Proof of Theorem 1.1-1.2}

Before proving Theorem 1.1, let us introduce some preliminary results. First, the following Hardy-LittlewoodSobolev inequality will be frequently used in the sequel.

Lemma 2.1 (Hardy-Littlewood-Sobolev inequality [10, Theorem 4.3]). Let $s, r>1$ and $0<\alpha<N$ with $1 / s+$ $1 / r=1+\alpha / N, f \in L^{s}\left(\mathbb{R}^{N}\right)$ and $g \in L^{r}\left(\mathbb{R}^{N}\right)$, then there exists a positive constant $C(s, N, \alpha)$ (independent of $f, g$ ) such that

$$
\left|\int_{\mathbb{R}^{N}} \int_{\mathbb{R}^{N}} f(x)\right| x-\left.y\right|^{\alpha-N} g(y) \mathrm{d} x \mathrm{~d} y \mid \leq C(s, N, \alpha)\|f\|_{s}\|g\|_{r} .
$$

In particular, if $s=r=2 N /(N+\alpha)$, the sharp constant is given by

$$
\mathcal{C}_{\alpha}:=\pi^{\frac{N-\alpha}{2}} \frac{\Gamma(\alpha / 2)}{\Gamma((N+\alpha) / 2)}\left[\frac{\Gamma(N / 2)}{\Gamma(N)}\right]^{-\alpha / N}
$$

Due to the presence of the lower critical exponent $\frac{N+\alpha}{N}$, the compactness fails in general. In fact, the convolution term enjoys the invariance of dilation, that is, for any $u \in L^{2}\left(\mathbb{R}^{N}\right)$ and $t>0$, one has

$$
G_{\infty}\left(u_{t}\right)=G_{\infty}(u), u_{t}(\cdot)=t^{\frac{N}{2}} u(t \cdot),
$$

where

$$
G_{\infty}(u)=\int_{\mathbb{R}^{N}}\left(I_{\alpha} \star|u|^{\frac{N+\alpha}{N}}\right)|u|^{\frac{N+\alpha}{N}} \mathrm{~d} x .
$$

To recover the compactness, the following Brezis-Lieb type lemma plays a crucial role in the decomposition of the maximization sequence for $c \star$ given below.

For any $u \in H^{1}\left(\mathbb{R}^{N}\right)$, let

$$
\begin{gathered}
G(u)=\int_{\mathbb{R}^{N}}\left(I_{\alpha} \star\left[Q(x)|u|^{\frac{N+\alpha}{N}}\right]\right) Q(x)|u|^{\frac{N+\alpha}{N}} \mathrm{~d} x, \\
T(u)=\int_{\mathbb{R}^{N}}\left(|\nabla u|^{2}+V(x)|u|^{2}\right) \mathrm{d} x .
\end{gathered}
$$


Lemma 2.2 (Brezis-Lieb type Lemma). Assume that $\left(V_{1}\right)$ and $\left(Q_{1}\right)$ hold and let $\left\{u_{n}\right\}_{n=1}^{\infty}$ be a bounded sequence in $L^{2}\left(\mathbb{R}^{N}\right)$ and for some $u \in L^{2}\left(\mathbb{R}^{N}\right), u_{n} \rightarrow u$ strongly in $L_{\text {loc }}^{2}\left(\mathbb{R}^{N}\right)$ as $n \rightarrow \infty$, then, up to a subsequence, there holds

$$
\lim _{n \rightarrow \infty}\left[G\left(u_{n}\right)-G(u)-G_{\infty}\left(u_{n}-u\right)\right]=0 .
$$

Proof. Without loss of generality, we may assume that $u_{n} \rightarrow u$ almost everywhere on $\mathbb{R}^{N}$ as $n \rightarrow \infty$. Let $v_{n}=\sqrt{Q} u_{n}, v=\sqrt{Q} u$, it follows from [19, Lemma 2.4] that

$$
\lim _{n \rightarrow \infty}\left[G\left(u_{n}\right)-G(u)-G\left(u_{n}-u\right)\right]=0 .
$$

In the following, we show that

$$
\lim _{n \rightarrow \infty}\left[G\left(u_{n}-u\right)-G_{\infty}\left(u_{n}-u\right)\right]=0
$$

Let $w_{n}=u_{n}-u$,

$$
\begin{aligned}
G\left(u_{n}-u\right)= & \int_{\mathbb{R}^{N}}\left(I_{\alpha} \star\left[Q(x)\left|w_{n}\right|^{\frac{N+\alpha}{N}}\right]\right) Q(x)\left|w_{n}\right|^{\frac{N+\alpha}{N}} \mathrm{~d} x \\
= & \int_{\mathbb{R}^{N}}\left(I_{\alpha} \star\left[Q(x)\left|w_{n}\right|^{\frac{N+\alpha}{N}}\right]\right)(Q(x)-1)\left|w_{n}\right|^{\frac{N+\alpha}{N}} \mathrm{~d} x \\
& +\int_{\mathbb{R}^{N}}\left(I_{\alpha} \star\left[(Q(x)-1)\left|w_{n}\right|^{\frac{N+\alpha}{N}}\right]\right)\left|w_{n}\right|^{\frac{N+\alpha}{N}} \mathrm{~d} x+G_{\infty}\left(w_{n}\right) \\
:= & J_{1, n}+J_{2, n}+G_{\infty}\left(w_{n}\right) .
\end{aligned}
$$

By virtue of Lemma 2.1, we have

$$
J_{1, n} \leq \mathcal{C}_{\alpha} \max _{x \in \mathbb{R}^{N}} Q(x)\left(\int_{\mathbb{R}^{N}}\left|w_{n}\right|^{2} \mathrm{~d} x\right)^{\frac{N+\alpha}{2 N}}\left(\int_{\mathbb{R}^{N}}|Q(x)-1|^{\frac{2 N}{N+\alpha}}\left|w_{n}\right|^{2} \mathrm{~d} x\right)^{\frac{N+\alpha}{2 N}} .
$$

Since $Q(x) \rightarrow 1$ as $|x| \rightarrow \infty$ and $w_{n} \rightarrow 0$ strongly in $L_{\text {loc }}^{2}\left(\mathbb{R}^{N}\right)$ as $n \rightarrow \infty$, one can get that

$$
\lim _{n \rightarrow \infty} \int_{\mathbb{R}^{N}}|Q(x)-1|^{\frac{2 N}{N+\alpha}}\left|w_{n}\right|^{2} \mathrm{~d} x=0,
$$

which implies that $J_{1, n} \rightarrow 0$ as $n \rightarrow \infty$. Similarly, we have $J_{2, n} \rightarrow 0$ as $n \rightarrow \infty$. The proof is complete.

Set

$$
c_{\infty}:=\sup \left\{G_{\infty}(u): \int_{\mathbb{R}^{N}}|u|^{2} \mathrm{~d} x=1, u \in L^{2}\left(\mathbb{R}^{N}\right)\right\}
$$

and

$$
c_{\star}:=\sup \left\{G(u): T(u)=1, u \in H^{1}\left(\mathbb{R}^{N}\right)\right\} .
$$

Then by Lemma 2.1, $0<c_{\star}, c_{\infty}<\infty$ and it follows from [10, Theorem 4.3] that $c_{\infty}$ can be achieved by the family of functions

$$
U(x)=C \lambda^{\frac{N}{2}}\left(\lambda^{2}+|x|^{2}\right)^{-\frac{N}{2}},
$$

for some fixed $C>0$ and $\lambda \in \mathbb{R}^{+}$as parameters.

Lemma 2.3 (Compactness). If $c_{\star}>c_{\infty}$, then $c_{\star}$ can be achieved.

Proof. For any minimization sequence $\left\{u_{n}\right\}_{n=1}^{\infty} \subset H^{1}\left(\mathbb{R}^{N}\right)$ of $c_{\star}$, i. e, $G\left(u_{n}\right) \rightarrow c_{\star}, \quad n \rightarrow \infty$ with

$$
\int_{\mathbb{R}^{N}}\left(\left|\nabla u_{n}\right|^{2}+V(x)\left|u_{n}\right|^{2}\right) \mathrm{d} x=1,
$$


without loss of generality, we assume that $u_{n}$ is non-negative for all $n$ and for some $u_{0} \in H^{1}\left(\mathbb{R}^{N}\right), u_{n} \rightarrow u_{0} \geq 0$ weakly in $H^{1}\left(\mathbb{R}^{N}\right)$, strongly in $L_{\text {loc }}^{2}\left(\mathbb{R}^{N}\right)$ and a. e. on $\mathbb{R}^{N}$ as $n \rightarrow \infty$. Thanks to Lemma 2.2,

$$
c_{\star}=G\left(u_{0}\right)+G_{\infty}\left(u_{n}-u_{0}\right)+o_{n}(1),
$$

where $o_{n}(1) \rightarrow 0$ as $n \rightarrow \infty$. Moreover, set $w_{n}=u_{n}-u_{0}$, we have

$$
1=\int_{\mathbb{R}^{N}}\left(\left|\nabla u_{0}\right|^{2}+V(x)\left|u_{0}\right|^{2}\right) \mathrm{d} x+\int_{\mathbb{R}^{N}}\left(\left|\nabla w_{n}\right|^{2}+\left|w_{n}\right|^{2}\right) \mathrm{d} x+o_{n}(1) .
$$

On the other hand, by the definitions of $c \star$ and $c_{\infty}$, it is easy to know that

$$
G(u) \leq c_{\star}\left(\int_{\mathbb{R}^{N}}\left(|\nabla u|^{2}+V(x)|u|^{2}\right) \mathrm{d} x\right)^{\frac{N+\alpha}{N}} \quad \text { for any } u \in H^{1}\left(\mathbb{R}^{N}\right)
$$

and

$$
G_{\infty}(u) \leq c_{\infty}\left(\int_{\mathbb{R}^{N}}|u|^{2} \mathrm{~d} x\right)^{\frac{N+\alpha}{N}}, \text { for any } u \in L^{2}\left(\mathbb{R}^{N}\right) .
$$

Then by (2.1) and (2.2),

$$
\begin{aligned}
c_{\star} & \leq c_{\star}\left(\int_{\mathbb{R}^{N}}\left(\left|\nabla u_{0}\right|^{2}+V(x)\left|u_{0}\right|^{2}\right) \mathrm{d} x\right)^{\frac{N+\alpha}{N}}+c_{\infty}\left(\int_{\mathbb{R}^{N}}\left|w_{n}\right|^{2} \mathrm{~d} x\right)^{\frac{N+\alpha}{N}}+o_{n}(1) \\
& \leq c_{\star}\left(\int_{\mathbb{R}^{N}}\left(\left|\nabla u_{0}\right|^{2}+V(x)\left|u_{0}\right|^{2}\right) \mathrm{d} x\right)^{\frac{N+\alpha}{N}}+c_{\infty}\left(\int_{\mathbb{R}^{N}}\left(\left|\nabla w_{n}\right|^{2}+\left|w_{n}\right|^{2}\right) \mathrm{d} x\right)^{\frac{N+\alpha}{N}}+o_{n}(1) \\
& \leq c_{\star} \int_{\mathbb{R}^{N}}\left(\left|\nabla u_{0}\right|^{2}+V(x)\left|u_{0}\right|^{2}\right) \mathrm{d} x+c_{\infty} \int_{\mathbb{R}^{N}}\left(\left|\nabla w_{n}\right|^{2}+\left|w_{n}\right|^{2}\right) \mathrm{d} x+o_{n}(1) .
\end{aligned}
$$

If $c_{\star}>c_{\infty}$, we claim that $w_{n} \rightarrow 0$ strongly in $H^{1}\left(\mathbb{R}^{N}\right)$ as $n \rightarrow \infty$. Otherwise, we have

$$
c_{\star}<c_{\star} \int_{\mathbb{R}^{N}}\left(\left|\nabla u_{0}\right|^{2}+V(x)\left|u_{0}\right|^{2}\right) \mathrm{d} x+c_{\star} \int_{\mathbb{R}^{N}}\left(\left|\nabla w_{n}\right|^{2}+\left|w_{n}\right|^{2}\right) \mathrm{d} x+o_{n}(1)=c_{\star}+o_{n}(1),
$$

which is a contradiction. So $w_{n} \rightarrow 0$ strongly in $H^{1}\left(\mathbb{R}^{N}\right)$ as $n \rightarrow \infty$ and then $u_{n} \rightarrow u_{0}$ strongly in $H^{1}\left(\mathbb{R}^{N}\right)$ as $n \rightarrow \infty$. This implies that $u_{0}$ is a non-negative maximizer of $c_{\star}$. The proof is complete.

In the following, we give a lower bound estimate for $c_{*}$. For any $\varepsilon>0$, set

$$
u_{\varepsilon}(x)=\varepsilon^{\frac{N}{2}} U(\varepsilon x),
$$

where $U(x)$ is a maximizer of $c_{\infty}$ and given above with $\lambda=1$. Following [20], we have

Lemma 2.4 (Energy estimate). Assume $\left(V_{1}\right),\left(V_{2}\right),\left(Q_{1}\right),\left(Q_{2}\right)$ hold, then $c_{*}>c_{\infty}$ if one of the following conditions holds

(i) $\beta=0, \mu>\frac{N^{2}(N-2)}{4(N+1)}, \inf _{x \in \mathbb{R}^{N}} Q(x) \geq 1$;

(ii) $0<\beta<2, v_{\beta}>0$;

(iii) $\beta>2, \mu>\frac{N^{2}(N-2)}{4(N+1)}$.

Proof. Observe that for any $\varepsilon>0$,

$$
\int_{\mathbb{R}^{N}}\left(I_{\alpha} \star\left|u_{\varepsilon}\right|^{\frac{N+\alpha}{N}}\right)\left|u_{\varepsilon}\right|^{\frac{N+\alpha}{N}}=\int_{\mathbb{R}^{N}}\left(I_{\alpha} \star|U|^{\frac{N+\alpha}{N}}\right)|U|^{\frac{N+\alpha}{N}}=c_{\infty},
$$




$$
\int_{\mathbb{R}^{N}}\left|u_{\varepsilon}\right|^{2}=\int_{\mathbb{R}^{N}}|U|^{2}=1, \int_{\mathbb{R}^{N}}\left|\nabla u_{\varepsilon}\right|^{2}=\varepsilon^{2} \int_{\mathbb{R}^{N}}|\nabla U|^{2}<+\infty .
$$

Let

$$
m_{\varepsilon}:=\int_{\mathbb{R}^{N}}\left|\nabla u_{\varepsilon}\right|^{2}+V(x)\left|u_{\varepsilon}\right|^{2}
$$

then $m_{\varepsilon}=1+\varepsilon^{2} \mathcal{J}_{\mu}(\varepsilon)$, where

$$
\mathcal{J}_{\mu}(\varepsilon)=\varepsilon^{-2} \int_{\mathbb{R}^{N}}\left[\left|\nabla u_{\varepsilon}(x)\right|^{2}+(V(x)-1)\left|u_{\varepsilon}(x)\right|^{2}\right] \mathrm{d} x .
$$

Since

$$
\begin{gathered}
\int_{\mathbb{R}^{N}}\left|\nabla u_{\varepsilon}\right|^{2}=\frac{N^{2}(N-2)}{4(N+1)} \int_{\mathbb{R}^{N}} \frac{\left|u_{\varepsilon}(x)\right|^{2}}{|x|^{2}} \mathrm{~d} x, \\
\mathcal{J}_{\mu}(\varepsilon)=\varepsilon^{-2} \int_{\mathbb{R}^{N}}\left[\left|\nabla u_{\varepsilon}(x)\right|^{2}+(V(x)-1)\left|u_{\varepsilon}(x)\right|^{2}\right] \mathrm{d} x .
\end{gathered}
$$

By Lebesgue's monotone convergence theorem, we obtain

$$
\begin{aligned}
\mathcal{J}_{\mu}(\varepsilon) & =\int_{\mathbb{R}^{N}}\left[\frac{N^{2}(N-2)}{4(N+1)}+\varepsilon^{-2}\left(V\left(\varepsilon^{-1} x\right)-1\right)|x|^{2}\right] \frac{|U(x)|^{2}}{|x|^{2}} \mathrm{~d} x \\
& \rightarrow\left[\frac{N^{2}(N-2)}{4(N+1)}-\mu\right] \int_{\mathbb{R}^{N}} \frac{|U(x)|^{2}}{|x|^{2}} \mathrm{~d} x \text {, as } \varepsilon \rightarrow 0 .
\end{aligned}
$$

Let

$$
a_{\mu}:=\left[\frac{N^{2}(N-2)}{4(N+1)}-\mu\right] \int_{\mathbb{R}^{N}} \frac{|U(x)|^{2}}{|x|^{2}} \mathrm{~d} x,
$$

then we get that

$$
m_{\varepsilon}=1+a_{\mu} \varepsilon^{2}+o\left(\varepsilon^{2}\right) \text {, as } \varepsilon \rightarrow 0
$$

and

$$
a_{\mu} \begin{cases}>0, & \text { if } \mu<\frac{N^{2}(N-2)}{4(N+1)} \\ =0, & \text { if } \mu=\frac{N^{2}(N-2)}{4(N+1)} \\ <0, & \text { if } \mu>\frac{N^{2}(N-2)}{4(N+1)} .\end{cases}
$$

Let $v_{\varepsilon}:=\frac{u_{\varepsilon}}{\sqrt{m_{\varepsilon}}}$, then

$$
\int_{\mathbb{R}^{N}}\left|\nabla v_{\varepsilon}\right|^{2}+V(x)\left|v_{\varepsilon}\right|^{2}=1
$$

and $c_{\star} \geq G\left(v_{\varepsilon}\right)$. In the following, we show that $G\left(v_{\varepsilon}\right)>c_{\infty}$ for $\varepsilon>0$ small. In fact,

$$
\begin{aligned}
m_{\varepsilon}^{\frac{N+\alpha}{N}} G\left(v_{\varepsilon}\right) & =\int_{\mathbb{R}^{N}}\left(I_{\alpha} \star\left[Q(x)\left|u_{\varepsilon}\right|^{\frac{N+\alpha}{N}}\right]\right) Q(x)\left|u_{\varepsilon}\right|^{\frac{N+\alpha}{N}} \mathrm{~d} x \\
& =\int_{\mathbb{R}^{N}}\left(I_{\alpha} \star\left[Q\left(\varepsilon^{-1} x\right)|U|^{\frac{N+\alpha}{N}}\right]\right) Q\left(\varepsilon^{-1} x\right)|U|^{\frac{N+\alpha}{N}} \mathrm{~d} x \\
& =\int_{\mathbb{R}^{N}}\left(I_{\alpha} \star\left[Q\left(\varepsilon^{-1} x\right)|U|^{\frac{N+\alpha}{N}}\right]\right)\left[Q\left(\varepsilon^{-1} x\right)-1\right]|U|^{\frac{N+\alpha}{N}} \mathrm{~d} x \\
& +\int_{\mathbb{R}^{N}}\left(I_{\alpha} \star\left[\left(Q\left(\varepsilon^{-1} x\right)-1\right)|U|^{\frac{N+\alpha}{N}}\right]\right)|U|^{\frac{N+\alpha}{N}} \mathrm{~d} x+G_{\infty}(U) .
\end{aligned}
$$


By virtue of Lemma 2.1, it is easy to check that

$$
\int_{\mathbb{R}^{N}}\left(I_{\alpha}{ }^{\star}\left[|x|^{-\beta}|U|^{\frac{N+\alpha}{N}}\right]\right)|U|^{\frac{N+\alpha}{N}} \mathrm{~d} x<\infty, \text { if } \beta \in[0, N) .
$$

It follows from the Lebesgue's monotone convergence theorem that, if $\beta \in[0, N)$,

$$
\begin{aligned}
& \lim _{\varepsilon \rightarrow 0} \varepsilon^{-\beta} \int_{\mathbb{R}^{N}}\left(I_{\alpha} \star\left[\left(Q\left(\varepsilon^{-1} x\right)-1\right)|U|^{\frac{N+\alpha}{N}}\right]\right)|U|^{\frac{N+\alpha}{N}} \mathrm{~d} x \\
& =v_{\beta} \int_{\mathbb{R}^{N}}\left(I_{\alpha} \star\left[|x|^{-\beta}|U|^{\frac{N+\alpha}{N}}\right]\right)|U|^{\frac{N+\alpha}{N}} \mathrm{~d} x .
\end{aligned}
$$

Similarly, if $\beta \in[0, N)$,

$$
\begin{aligned}
& \lim _{\varepsilon \rightarrow 0} \varepsilon^{-\beta} \int_{\mathbb{R}^{N}}\left(I_{\alpha} \star\left[Q\left(\varepsilon^{-1} x\right)|U|^{\frac{N+\alpha}{N}}\right]\right)\left[Q\left(\varepsilon^{-1} x\right)-1\right]|U|^{\frac{N+\alpha}{N}} \mathrm{~d} x \\
& =v_{\beta} \int_{\mathbb{R}^{N}}\left(\left.I_{\alpha} \star U\right|^{\frac{N+\alpha}{N}}\right)\left[|x|^{-\beta}|U|^{\frac{N+\alpha}{N}}\right] \mathrm{d} x .
\end{aligned}
$$

Let

$$
b_{\beta, \alpha}:=2 \int_{\mathbb{R}^{N}}\left(I_{\alpha}{ }^{\star}|U|^{\frac{N+\alpha}{N}}\right)\left[|x|^{-\beta}|U|^{\frac{N+\alpha}{N}}\right] \mathrm{d} x>0,
$$

then, for $\beta \in[0, N)$, we have, as $\varepsilon \rightarrow 0$,

$$
\begin{aligned}
G\left(v_{\varepsilon}\right) & =m_{\varepsilon}^{-\frac{N+\alpha}{N}}\left(c_{\infty}+b_{\beta, \alpha} v_{\beta} \varepsilon^{\beta}+o\left(\varepsilon^{\beta}\right)\right) \\
& =\left(1+a_{\mu} \varepsilon^{2}+o\left(\varepsilon^{2}\right)\right)^{-\frac{N+\alpha}{N}}\left(c_{\infty}+b_{\beta, \alpha} v_{\beta} \varepsilon^{\beta}+o\left(\varepsilon^{\beta}\right)\right) \\
& =\left(1-\frac{N+\alpha}{N} a_{\mu} \varepsilon^{2}+o\left(\varepsilon^{2}\right)\right)\left(c_{\infty}+b_{\beta, \alpha} v_{\beta} \varepsilon^{\beta}+o\left(\varepsilon^{\beta}\right)\right) \\
& =c_{\infty}+b_{\beta, \alpha} v_{\beta} \varepsilon^{\beta}-\frac{N+\alpha}{N} c_{\infty} a_{\mu} \varepsilon^{2}+o\left(\varepsilon^{\beta}\right)+o\left(\varepsilon^{2}\right) .
\end{aligned}
$$

which implies that if $2<\beta<N$,

$$
G\left(v_{\varepsilon}\right)=c_{\infty}-\frac{N+\alpha}{N} c_{\infty} a_{\mu} \varepsilon^{2}+o\left(\varepsilon^{2}\right), \text { as } \varepsilon \rightarrow 0 .
$$

Moveover, by $\left(Q_{1}\right)$, if $\beta=0$ and in addition $\inf _{x \in \mathbb{R}^{N}} Q(x) \geq 1$, we know $v_{\beta}=0$ and

$$
G\left(v_{\varepsilon}\right) \geq m_{\varepsilon}^{-\frac{N+\alpha}{N}} c_{\infty} \geq c_{\infty}-\frac{N+\alpha}{N} c_{\infty} a_{\mu} \varepsilon^{2}+o\left(\varepsilon^{2}\right), \text { as } \varepsilon \rightarrow 0 .
$$

It follows from (2.3) and (2.4) that $G\left(v_{\varepsilon}\right)>c_{\infty}$ for $\varepsilon>0$ small if

$$
\mu>\frac{N^{2}(N-2)}{4(N+1)}, \beta=0 \text { or } 2<\beta<N .
$$

For $\beta \geq N$, by $\left(Q_{2}\right)$ we have for some $C>0$,

$$
|x|^{2}|Q(x)-1| \leq \frac{C}{|x|^{\beta-2}}, x \in \mathbb{R} \backslash\{0\} .
$$

Noting that

$$
\int_{\mathbb{R}^{N}}\left(I_{\alpha} \star\left[|x|^{-2}|U|^{\frac{N+\alpha}{N}}\right]\right)|U|^{\frac{N+\alpha}{N}} \mathrm{~d} x<\infty
$$


due to the Lebesgue's monotone convergence theorem, if $\beta \geq N$,

$$
\lim _{\varepsilon \rightarrow 0} \varepsilon^{-2} \int_{\mathbb{R}^{N}}\left(I_{\alpha} \star\left[\left(Q\left(\varepsilon^{-1} \chi\right)-1\right)|U|^{\frac{N+\alpha}{N}}\right]\right)|U|^{\frac{N+\alpha}{N}} \mathrm{~d} x=0 .
$$

Similarly, if $\beta \geq N$,

$$
\lim _{\varepsilon \rightarrow 0} \varepsilon^{-2} \int_{\mathbb{R}^{N}}\left(I_{\alpha} \star\left[Q\left(\varepsilon^{-1} \chi\right)|U|^{\frac{N+\alpha}{N}}\right]\right)\left[Q\left(\varepsilon^{-1} \chi\right)-1\right]|U|^{\frac{N+\alpha}{N}} \mathrm{~d} x=0 .
$$

This yields that, for $\beta \geq N$, as $\varepsilon \rightarrow 0$,

$$
\begin{aligned}
G\left(v_{\varepsilon}\right) & =m_{\varepsilon}^{-\frac{N+\alpha}{N}}\left(c_{\infty}+o\left(\varepsilon^{2}\right)\right) \\
& =\left(1+a_{\mu} \varepsilon^{2}+o\left(\varepsilon^{2}\right)\right)^{-\frac{N+\alpha}{N}}\left(c_{\infty}+o\left(\varepsilon^{2}\right)\right) \\
& =\left(1-\frac{N+\alpha}{N} a_{\mu} \varepsilon^{2}+o\left(\varepsilon^{2}\right)\right)\left(c_{\infty}+o\left(\varepsilon^{2}\right)\right) \\
& =c_{\infty}-\frac{N+\alpha}{N} c_{\infty} a_{\mu} \varepsilon^{2}+o\left(\varepsilon^{2}\right),
\end{aligned}
$$

As a consequence, $G\left(v_{\varepsilon}\right)>c_{\infty}$ for $\varepsilon>0$ small if

$$
\mu>\frac{N^{2}(N-2)}{4(N+1)}, \beta \geq N
$$

Finally, if $0<\beta<2$, then

$$
G\left(v_{\varepsilon}\right)=c_{\infty}+b_{\beta, \alpha} v_{\beta} \varepsilon^{\beta}+o\left(\varepsilon^{\beta}\right) \text {, as } \varepsilon \rightarrow 0,
$$

which implies that $G\left(v_{\varepsilon}\right)>c_{\infty}$ for $\varepsilon>0$ small and any $\mu \in \mathbb{R}, v_{\beta}>0$. The proof is complete.

\section{Proof of Theorem 1.1 completed}

Proof. As an immediate consequence of Lemma 2.3 and 2.4 , there exists $u_{\star} \in H^{1}\left(\mathbb{R}^{N}\right)$ such that $G\left(u_{\star}\right)=c_{\star}$ and

$$
\int_{\mathbb{R}^{N}}\left(|\nabla u \star|^{2}+V(x)|u \star|^{2}\right) \mathrm{d} x=1
$$

By the Lagrange multiplier theorem, there holds that for some $\kappa \in \mathbb{R}$ such that $G^{\prime}\left(u_{\star}\right)=\kappa T^{\prime}(u)$ in $H^{-1}\left(\mathbb{R}^{N}\right)$, that is, in the weak sense, $u \star$ satisfies

$$
\kappa(-\Delta u+V(x) u)=\frac{N+\alpha}{N}\left(I_{\alpha} \star\left[Q(x)|u|^{\frac{N+\alpha}{N}}\right]\right) Q(x)|u|^{\frac{\alpha}{N}-1} u, \quad x \in \mathbb{R}^{N} .
$$

Obviously, $\kappa=\frac{N+\alpha}{N} c_{\star}>0$ and by virtue of the maximum principle, $u_{\star}$ is positive. To remove the multiplier, let

$$
u_{\theta}(\cdot)=\theta u \star(\cdot), \theta=c_{\star}^{-\frac{N}{2 \alpha}},
$$

then $u_{\theta}$ is a weak solution of problem (1.8) and

$$
\begin{aligned}
I\left(u_{\theta}\right) & =\frac{1}{2} T\left(u_{\theta}\right)-\frac{N}{2(N+\alpha)} G\left(u_{\theta}\right) \\
& =\frac{1}{2} \theta^{2} T\left(u_{\star}\right)-\frac{N}{2(N+\alpha)} \theta^{\frac{2(N+\alpha)}{N}} G\left(u_{\star}\right) \\
& =\frac{1}{2} \theta^{2}-\frac{N}{2(N+\alpha)} \theta^{\frac{2(N+\alpha)}{N}} c_{\star}=\frac{\alpha}{2(N+\alpha)} c_{\star}^{-\frac{N}{\alpha}}>0 .
\end{aligned}
$$

Finally, we show that $u_{\theta}$ is a ground state solution of problem (1.8). In fact, for any nontrivial solution $u$ of problem (1.8), we can see that $T(u)=G(u)>0$ and

$$
I(u)=\frac{\alpha}{2(N+\alpha)} G(u) .
$$


Let

then $T\left(u_{\tau}\right)=1$ and

$$
\tau=\sqrt{\frac{1}{T(u)}}>0, u_{\tau}(\cdot)=\tau u(\cdot)
$$

$$
c \star \geq G\left(u_{\tau}\right)=\tau^{\frac{2(N+\alpha)}{N}} G(u) .
$$

It follows that

$$
G(u) \leq C_{\star}[T(u)]^{\frac{N+\alpha}{N}}=C_{\star}[G(u)]^{\frac{N+\alpha}{N}}
$$

and then

$$
I(u) \geq \frac{\alpha}{2(N+\alpha)} c_{\star}^{-\frac{N}{\alpha}} .
$$

The proof is complete.

Proposition 2.1 (Pohozăev Identity). If $u \in H^{1}\left(\mathbb{R}^{N}\right)$ is a solution of problem (1.8), then the following Pohozăev identity holds

$$
\begin{aligned}
& \frac{N-2}{2} \int_{\mathbb{R}^{N}}|\nabla u|^{2} \mathrm{~d} x+\frac{1}{2} \int_{\mathbb{R}^{N}}[N V(x)+\langle x, \nabla V(x)\rangle] u^{2} \mathrm{~d} x \\
& =\frac{N}{2} \int_{\mathbb{R}^{N}}\left(I_{\alpha} \star\left[Q(x)|u|^{\frac{N+\alpha}{N}}\right]\right) Q(x)|u|^{\frac{N+\alpha}{N}} \mathrm{~d} x \\
& +\frac{N}{N+\alpha} \int_{\mathbb{R}^{N}}\left(I_{\alpha}{ }^{\star}\left[Q(x)|u|^{\frac{N+\alpha}{N}}\right]\right)\langle x, \nabla Q(x)\rangle|u|^{\frac{N+\alpha}{N}} \mathrm{~d} x .
\end{aligned}
$$

Proof. The proof is similar to [20, Proposition 11] and [22, Theorem 3]. We omit the details here.

Completion of Proof of Theorem 1.2

Proof. For any solution $u \in H^{1}\left(\mathbb{R}^{N}\right)$ of problem (1.8), using $u$ as a test function, we have

$$
\int_{\mathbb{R}^{N}}\left(|\nabla u|^{2}+V(x)|u|^{2}\right) \mathrm{d} x=\int_{\mathbb{R}^{N}}\left(I_{\alpha}{ }^{\star}\left[Q(x)|u|^{\frac{N+\alpha}{N}}\right]\right) Q(x)|u|^{\frac{N+\alpha}{N}} \mathrm{~d} x
$$

Thanks to Proposition 2.1,

$$
\begin{aligned}
& \int_{\mathbb{R}^{N}}\left(-|\nabla u|^{2}+\frac{1}{2}\langle x, \nabla V(x)\rangle u^{2}\right) \mathrm{d} x \\
& =\frac{N}{N+\alpha} \int_{\mathbb{R}^{N}}\left(I_{\alpha} \star\left[Q(x)|u|^{\frac{N+\alpha}{N}}\right]\right)\langle x, \nabla Q(x)\rangle|u|^{\frac{N+\alpha}{N}} \mathrm{~d} x .
\end{aligned}
$$

Then by $(V Q)$ and Hardy's inequality, if $u$ is nontrivial,

$$
\int_{\mathbb{R}^{N}}|\nabla u|^{2} \mathrm{~d} x<\frac{(N-2)^{2}}{4} \int_{\mathbb{R}^{N}} \frac{|u(x)|^{2}}{|x|^{2}} \mathrm{~d} x \leq \int_{\mathbb{R}^{N}}|\nabla u|^{2} \mathrm{~d} x,
$$

which is a contradiction. The proof is complete.

\section{Proof of Theorem 1.3}

\subsection{Eigenvalues and eigenfunctions.}

Consider the eigenvalue problem

$$
-\Delta u+u=\frac{\lambda}{v^{2}+|x|^{2}} u, u \in H^{1}\left(\mathbb{R}^{N}\right) .
$$


It was proven in [7] that problem (3.1) admits a sequence of eigenvalues $\left\{\lambda_{n}\right\}$ with finite multiplicity and associated eigenfunctions $\left\{\varphi_{n}\right\}$, such that

$$
0<\mu^{v}=\lambda_{1}<\lambda_{2} \leq \cdots \leq \lambda_{n} \leq \cdots \rightarrow+\infty, n \rightarrow \infty
$$

and for any $i, j \in \mathbb{N}$ and $i \neq j$,

$$
\int_{\mathbb{R}^{N}} \nabla \varphi_{i} \nabla \varphi_{j}+\varphi_{i} \varphi_{j}=0, \int_{\mathbb{R}^{N}} \frac{1}{v^{2}+|x|^{2}}\left|\varphi_{i}\right|^{2} \mathrm{~d} x=1 .
$$

Moreover, for some $C_{n}, \delta_{n}>0$,

$$
\left|\varphi_{n}(x)\right|+\left|\nabla \varphi_{n}(x)\right| \leq C_{n} \exp \left(-\delta_{n}|x|\right), \quad x \in \mathbb{R}^{N} .
$$

For any $n \in \mathbb{N}$, we have the orthogonal decomposition $H^{1}\left(\mathbb{R}^{N}\right)=E^{-} \oplus E^{+}$, where

$$
E^{-}=\operatorname{span}\left\{\varphi_{1}, \varphi_{2}, \cdots, \varphi_{n}\right\}, \quad E^{+}=\overline{\operatorname{span}\left\{\varphi_{n+1}, \varphi_{n+2}, \cdots\right\}} .
$$

\subsection{Energy estimates.}

For any $\varepsilon>0$, set

$$
u_{\varepsilon}(x)=\varepsilon^{\frac{N}{2}} U(\varepsilon x),
$$

where $U$ is given in Section 2 above for $\lambda=v$. Similarly, we have

$$
\int_{\mathbb{R}^{N}}\left(I_{\alpha} \star\left|u_{\varepsilon}\right|^{\frac{N+\alpha}{N}}\right)\left|u_{\varepsilon}\right|^{\frac{N+\alpha}{N}}=c_{\infty}
$$

and

$$
m_{\varepsilon}:=\int_{\mathbb{R}^{N}}\left|\nabla u_{\varepsilon}\right|^{2}+V_{\mu, v}(x)\left|u_{\varepsilon}\right|^{2}=1+a_{\mu, v} \varepsilon^{2}+o\left(\varepsilon^{2}\right), \text { as } \varepsilon \rightarrow 0
$$

where

$$
a_{\mu, v}:=C v^{-2}\left[\frac{N^{2}(N-2)}{4(N+1)}-\mu\right] \int_{\mathbb{R}^{N}} \frac{1}{|x|^{2}\left(1+|x|^{2}\right)^{N}} \mathrm{~d} x
$$

and $C>0$ is independent of $\varepsilon, \mu, v$.

From now on, we assume

$$
\mu>\max \left\{\frac{N^{2}(N-2)}{4(N+1)}, \mu^{v}\right\}
$$

and for some $n \in \mathbb{N}$, we assume $\mu \in\left[\lambda_{n}, \lambda_{n+1}\right)$ when $N \geq 4$ or $\mu \in\left(\lambda_{n}, \lambda_{n+1}\right)$ when $N=3$. Define

$$
\hat{E}\left(v_{\varepsilon}\right):=\left\{w \in H^{1}\left(\mathbb{R}^{N}\right): w=t v_{\varepsilon}+v, t \geq 0, v \in E^{-}\right\}
$$

where $v_{\varepsilon}:=\frac{u_{\varepsilon}}{\sqrt{m_{\varepsilon}}}$ and the energy functional

$$
\begin{aligned}
J(u)= & \frac{1}{2} \int_{\mathbb{R}^{N}}\left(|\nabla u|^{2}+V_{\mu, v}(x)|u|^{2}\right) \mathrm{d} x \\
& -\frac{N}{2(N+\alpha)} \int_{\mathbb{R}^{N}}\left(I_{\alpha} \star\left[Q_{\beta}(x)|u|^{\frac{N+\alpha}{N}}\right]\right) Q_{\beta}(x)|u|^{\frac{N+\alpha}{N}} \mathrm{~d} x .
\end{aligned}
$$

Lemma 3.1. For $\varepsilon>0$ small enough, there holds that

$$
\sup _{w \in \hat{E}\left(v_{\varepsilon}\right)} J(w)<\frac{\alpha}{2(N+\alpha)} c_{\infty}^{-\frac{N}{\alpha}},
$$

provided when $N=3$, one of the followings holds 
(i) $\beta=0, v_{\beta}=0, \frac{3}{2}<\alpha<3$;

(ii) $0<\beta<\min \left\{\frac{9}{6-\alpha}, 2\right\}, v_{\beta}>0$

(iii) $\beta>2, v_{\beta}>-1, \frac{3}{2}<\alpha<3$;

and when $N \geq 4$, one of the followings holds

(iv) $\beta=0, v_{\beta}=0$;

(v) $0<\beta<2, v_{\beta}>0$;

(vi) $\beta>2, v_{\beta}>-1$.

Proof. For any $v \in E^{-}$and $t>0$,

$$
\begin{aligned}
& J\left(t v_{\varepsilon}+v\right) \\
& =\frac{t^{2}}{2} \int_{\mathbb{R}^{N}}\left|\nabla v_{\varepsilon}\right|^{2}+V_{\mu, v}\left|v_{\varepsilon}\right|^{2}+t \int_{\mathbb{R}^{N}} \nabla v_{\varepsilon} \cdot \nabla v+V_{\mu, v} v_{\varepsilon} v+\frac{1}{2} \int_{\mathbb{R}^{N}}|\nabla v|^{2}+V_{\mu, v}|v|^{2} \\
& \quad-\frac{N}{2(N+\alpha)} \int_{\mathbb{R}^{N}}\left(I_{\alpha} \star\left[Q_{\beta}(x)\left|t v_{\varepsilon}+v\right|^{\frac{N+\alpha}{N}}\right]\right) Q_{\beta}(x)\left|t v_{\varepsilon}+v\right|^{\frac{N+\alpha}{N}} .
\end{aligned}
$$

Noting that $v \in E^{-}, \int_{\mathbb{R}^{N}}|\nabla v|^{2}+V_{\mu, v}|v|^{2} \leq 0$ and then

$$
\begin{aligned}
J\left(t v_{\varepsilon}+v\right) \leq & \frac{t^{2}}{2}+t \int_{\mathbb{R}^{N}} \nabla v_{\varepsilon} \cdot \nabla v+V_{\mu, v} v_{\varepsilon} v \\
& -\frac{N}{2(N+\alpha)} \int_{\mathbb{R}^{N}}\left(I_{\alpha}{ }^{\star}\left[Q_{\beta}(x)\left|t v_{\varepsilon}+v\right|^{\frac{N+\alpha}{N}}\right]\right) Q_{\beta}(x)\left|t v_{\varepsilon}+v\right|^{\frac{N+\alpha}{N}} .
\end{aligned}
$$

Since $\left|\nabla v_{\varepsilon}\right| \leq C_{1} \varepsilon^{\frac{N}{2}+1}$ and $|\nabla v| \in L^{r}\left(\mathbb{R}^{N}\right)$ for any $r>0$,

$$
\left|\int_{\mathbb{R}^{N}} \nabla v_{\varepsilon} \cdot \nabla v+V_{\mu, \nu} v_{\varepsilon} v\right| \leq\left\|\nabla v_{\varepsilon}\right\|_{\infty}\|\nabla v\|_{L^{1}}+(1+\mu)\left\|v_{\varepsilon}\right\|_{\infty}\|v\|_{1} .
$$

Recalling that it is proven in [18] that

$$
\|u\|_{\star}:=\left[\int_{\mathbb{R}^{N}}\left(I_{\alpha}{ }^{\star}|u|^{\frac{N+\alpha}{N}}\right)|u|^{\frac{N+\alpha}{N}}\right]^{\frac{N}{2(N+\alpha)}}, u \in L^{2}\left(\mathbb{R}^{N}\right),
$$

is a norm in $L^{2}\left(\mathbb{R}^{N}\right)$. Thanks to the fact that $\operatorname{dim}\left(E^{-}\right)=n$ and all the norms are equivalent in $E^{-}$, we get that

$$
\left|\int_{\mathbb{R}^{N}} \nabla v_{\varepsilon} \cdot \nabla v+V_{\mu, \nu} v_{\varepsilon} v\right| \leq C_{2} \varepsilon^{\frac{N}{2}}\|v\| .
$$

In the following, we estimate the convolution term

$$
G\left(t v_{\varepsilon}+v\right):=\int_{\mathbb{R}^{N}}\left(I_{\alpha} \star\left[Q_{\beta}(x)\left|t v_{\varepsilon}+v\right|^{\frac{N+\alpha}{N}}\right]\right) Q_{\beta}(x)\left|t v_{\varepsilon}+v\right|^{\frac{N+\alpha}{N}} .
$$

By [7, Lemma 3.5], for any $x \in \mathbb{R}^{N}, \varepsilon>0, v \in E^{-}$and $t>0$,

$$
|| t v_{\varepsilon}+\left.v\right|^{\frac{N+\alpha}{N}}-\left|t v_{\varepsilon}\right|^{\frac{N+\alpha}{N}}-\left.|v|^{\frac{N+\alpha}{N}}\left|\leq C_{3}\right| t v_{\varepsilon}|| v\right|^{\frac{\alpha}{N}},
$$

where $C_{3}=2^{(N-\alpha) / N}(N+\alpha) / N$. It follows that

$$
\int_{\mathbb{R}^{N}}\left(I_{\alpha}{ }^{*}\left[Q_{\beta}(x)\left|t v_{\varepsilon}+v\right|^{\frac{N+\alpha}{N}}\right]\right) Q_{\beta}(x)\left|t v_{\varepsilon}+v\right|^{\frac{N+\alpha}{N}}
$$




$$
\begin{aligned}
\geq & t^{\frac{2(N+\alpha)}{N}} \int_{\mathbb{R}^{N}}\left(I_{\alpha} \star\left[Q_{\beta}(x)\left|v_{\varepsilon}\right|^{\frac{N+\alpha}{N}}\right]\right) Q_{\beta}(x)\left|v_{\varepsilon}\right|^{\frac{N+\alpha}{N}} \\
& +2 t^{\frac{N+\alpha}{N}} \int_{\mathbb{R}^{N}}\left(I_{\alpha} \star\left[Q_{\beta}(x)\left|v_{\varepsilon}\right|^{\frac{N+\alpha}{N}}\right]\right) Q_{\beta}(x)|v|^{\frac{N+\alpha}{N}} \\
& +\int_{\mathbb{R}^{N}}\left(I_{\alpha} \star\left[Q_{\beta}(x)|v|^{\frac{N+\alpha}{N}}\right]\right) Q_{\beta}(x)|v|^{\frac{N+\alpha}{N}} \\
& -2 C_{3} \int_{\mathbb{R}^{N}}\left(I_{\alpha} \star\left[Q_{\beta}(x)\left(\left|t v_{\varepsilon}\right|^{\frac{N+\alpha}{N}}+|v|^{\frac{N+\alpha}{N}}\right)\right]\right) Q_{\beta}(x)\left|t v_{\varepsilon}\right||v|^{\frac{\alpha}{N}} \\
:= & J_{1}+J_{2}+J_{3}+J_{4} .
\end{aligned}
$$

Obviously, $J_{2} \geq 0$. So combing (3.3)-(3.6), we have

$$
J\left(t v_{\varepsilon}+v\right) \leq \frac{1}{2} t^{2}+C_{2} t \varepsilon^{\frac{N}{2}}\|v\|-\frac{N}{2(N+\alpha)}\left(J_{1}+J_{3}+J_{4}\right) .
$$

Similarly as above, as $\varepsilon \rightarrow 0$,

$$
t^{-\frac{2(N+\alpha)}{N}} J_{1} \geq \begin{cases}c_{\infty}-\frac{N+\alpha}{N} c_{\infty} a_{\mu, \nu} \varepsilon^{2}+o\left(\varepsilon^{2}\right), & \text { if } \beta=0, v_{\beta}=0 ; \\ c_{\infty}+b_{\beta, \alpha} v_{\beta} \varepsilon^{\beta}+o\left(\varepsilon^{\beta}\right), & \text { if } 0<\beta<2, v_{\beta}>0 ; \\ c_{\infty}-\frac{N+\alpha}{N} c_{\infty} a_{\mu, \nu} \varepsilon^{2}+o\left(\varepsilon^{2}\right), & \text { if } \beta>2, v_{\beta}>-1\end{cases}
$$

Due to the equivalence of norms in $E^{-}, J_{3} \geq C_{4}\|v\|^{\frac{2(N+\alpha)}{N}}$. Thanks to the Hardy-Littlewood-Sobolev inequality and $Q_{\beta} \in L^{\infty}\left(\mathbb{R}^{N}\right)$,

$$
\left|J_{4}\right| \leq C_{5} t\left(\left\|t v_{\varepsilon}\right\|_{L^{2}}+\|v\|_{L^{2}}\right)^{\frac{N+\alpha}{N}}\left(\int_{\mathbb{R}^{N}}\left|v_{\varepsilon}\right|^{\frac{2 N}{N+\alpha}}|v|^{\frac{2 \alpha}{N+\alpha}}\right)^{\frac{N+\alpha}{2 N}} .
$$

Then noting that $\left|v_{\varepsilon}(x)\right| \leq C_{6} \varepsilon^{\frac{N}{2}}, x \in \mathbb{R}^{N}$ and $E^{-} \subset L^{\frac{2 \alpha}{N+\alpha}}\left(\mathbb{R}^{N}\right)$,

$$
\int_{\mathbb{R}^{N}}\left|v_{\varepsilon}\right|^{\frac{2 N}{N+\alpha}}|v|^{\frac{2 \alpha}{N+\alpha}} \leq C_{7} \varepsilon^{\frac{N^{2}}{N+\alpha}} \int_{\mathbb{R}^{N}}|v|^{\frac{2 \alpha}{N+\alpha}} .
$$

Thanks to $\operatorname{dim}\left(E^{-}\right)<\infty$, it is easy to check that

$$
\int_{\mathbb{R}^{N}}|v|^{\frac{2 \alpha}{N+\alpha}} \leq C_{8}\|v\|^{\frac{2 \alpha}{N+\alpha}}, \text { for all } v \in E^{-},
$$

which implies that

$$
\left|J_{4}\right| \leq C_{9}\left(t^{\frac{N+\alpha}{N}}\|v\|^{\frac{\alpha}{N}}+\|v\|^{\frac{N+2 \alpha}{N}}\right) t \varepsilon^{\frac{N}{2}} .
$$

Let $C_{10}=\frac{N C_{4}}{2(N+\alpha)}$ (independent of $v$ ), by Young's inequality the following hold:

$$
\begin{aligned}
C_{2} t \varepsilon^{\frac{N}{2}}\|v\| & \leq \frac{C_{10}}{3}\|v\|^{\frac{2(N+\alpha)}{N}}+C_{11} t^{\frac{2(N+\alpha)}{N+2 \alpha}} \varepsilon^{\frac{N(N+\alpha)}{N+2 \alpha}}, \\
C_{9}\|v\|^{\frac{\alpha}{N}} t^{\frac{2 N+\alpha}{N}} \varepsilon^{\frac{N}{2}} & \leq \frac{C_{10}}{3}\|v\|^{\frac{2(N+\alpha)}{N}}+C_{12} t^{\frac{2(N+\alpha)}{N}} \varepsilon^{\frac{N(N+\alpha)}{2 N+\alpha},} \\
C_{9}\|v\|^{\frac{N+2 \alpha}{N}} t \varepsilon^{\frac{N}{2}} & \leq \frac{C_{10}}{3}\|v\|^{\frac{2(N+\alpha)}{N}}+C_{13} t^{\frac{2(N+\alpha)}{N}} \varepsilon^{N+\alpha,},
\end{aligned}
$$

from which we obtain

$$
\left|J_{4}\right| \leq \frac{2 C_{10}}{3}\|v\|^{\frac{2(N+\alpha)}{N}}+C_{12} t^{\frac{2(N+\alpha)}{N}} \varepsilon^{\frac{N(N+\alpha)}{2 N+\alpha}}+C_{13} t^{\frac{2(N+\alpha)}{N}} \varepsilon^{N+\alpha} .
$$

Then it follows from (3.7)-(3.8) that

$$
\begin{aligned}
J\left(t v_{\varepsilon}+v\right) \leq & \frac{1}{2} t^{2}-\frac{N}{2(N+\alpha)} J_{1}+C_{11} t^{\frac{2(N+\alpha)}{N+2 \alpha}} \varepsilon^{\frac{N(N+\alpha)}{N+2 \alpha}} \\
& +\frac{N}{2(N+\alpha)} t^{\frac{2(N+\alpha)}{N}}\left(C_{12} \varepsilon^{\frac{N(N+\alpha)}{2 N+\alpha}}+C_{13} \varepsilon^{N+\alpha}\right) .
\end{aligned}
$$


In particular, (3.11) implies that there exist $0<t_{\star}<t^{\star}$ (independent of $\varepsilon$ ) such that, for all $v \in E^{-}, \varepsilon>0$ small,

$$
J\left(t v_{\varepsilon}+v\right) \leq \frac{\alpha}{4(N+\alpha)} c_{\infty}^{-\frac{N}{\alpha}}, t \in\left(0, t_{\star}\right) \cup\left(t^{\star}, \infty\right) .
$$

If $\mu \in\left(\lambda_{n}, \lambda_{n+1}\right)$, there exists a constant $C_{14}$ such that

$$
\int_{\mathbb{R}^{N}}|\nabla v|^{2}+V_{\mu, v} \leq-C_{14}\|v\|^{2}, \text { for all } v \in E^{-} .
$$

Similarly as above, by (3.2) we have in place of (3.7) that

$$
J\left(t v_{\varepsilon}+v\right) \leq \frac{1}{2} t^{2}-\frac{C_{14}}{2}\|v\|^{2}+C_{2} t \varepsilon^{\frac{N}{2}}\|v\|-\frac{N}{2(N+\alpha)}\left(J_{1}+J_{3}+J_{4}\right) .
$$

We now use again estimates (3.8) and (3.10) and (3.9) replaced by the following

$$
C_{9}\|v\|^{\frac{\alpha}{N}} t^{\frac{2 N+\alpha}{N}} \varepsilon^{\frac{N}{2}} \leq C_{14}\|v\|^{2}+C_{15} t^{\frac{2(2 N+\alpha)}{2 N-\alpha}} \varepsilon^{\frac{N^{2}}{2 N-\alpha}},
$$

to get the following estimate

$$
\begin{aligned}
J\left(t v_{\varepsilon}+v\right) \leq & \frac{1}{2} t^{2}-\frac{N}{2(N+\alpha)} J_{1}+C_{11} t^{\frac{2(N+\alpha)}{N+2 \alpha}} \varepsilon^{\frac{N(N+\alpha)}{N+2 \alpha}} \\
& +\frac{N}{2(N+\alpha)}\left(C_{15} t^{\frac{2(2 N+\alpha)}{2 N-\alpha}} \varepsilon^{\frac{N^{2}}{2 N-\alpha}}+C_{13} t^{\frac{2(N+\alpha)}{N}} \varepsilon^{N+\alpha}\right) .
\end{aligned}
$$

Case 1. $\beta=v_{\beta}=0$ or $\beta>2, v_{\beta} \geq-1$.

If $N \geq 4$, then $\frac{N(N+\alpha)}{2 N+\alpha}>2$. Moreover, it follows from (3.11) that, for all $v \in E^{-}$, as $\varepsilon \rightarrow 0$, there holds that

$$
\begin{aligned}
J\left(t v_{\varepsilon}+v\right) \leq \frac{1}{2} t^{2}-\frac{N}{2(N+\alpha)} t^{\frac{2(N+\alpha)}{N}} & \left(c_{\infty}-\frac{N+\alpha}{N} c_{\infty} a_{\mu, \nu} \varepsilon^{2}-C_{12} \varepsilon^{\frac{N(N+\alpha)}{2 N+\alpha}}\right. \\
& \left.+o\left(\varepsilon^{2}\right)+o\left(\varepsilon^{\frac{N(N+\alpha)}{2 N+\alpha}}\right)\right), t \in\left[t_{*}, t^{*}\right],
\end{aligned}
$$

which implies that

$$
J\left(t v_{\varepsilon}+v\right) \leq \frac{1}{2} t^{2}-\frac{N}{2(N+\alpha)} t^{\frac{2(N+\alpha)}{N}}\left(c_{\infty}-\frac{N+\alpha}{N} c_{\infty} a_{\mu, \nu} \varepsilon^{2}+o\left(\varepsilon^{2}\right)\right), t \in\left[t_{*}, t^{\star}\right] .
$$

Noting that

$$
\max _{t \geq 0} g(t)=g\left(c_{\infty}^{-\frac{N}{2 \alpha}}\right)=\frac{\alpha}{2(N+\alpha)} c_{\infty}^{-\frac{N}{\alpha}}
$$

where

$$
g(t)=\frac{1}{2} t^{2}-\frac{N}{2(N+\alpha)} t^{\frac{2(N+\alpha)}{N}} c_{\infty} .
$$

So thanks to (3.12) and $a_{\mu, v}<0$ when $\mu>\frac{N^{2}(N-2)}{4(N+1)}$, we have as $\varepsilon>0$ small,

$$
\sup _{w \in \hat{E}\left(v_{\varepsilon}\right)} J(w) \leq \max _{t \geq 0} g(t)+C_{16} c_{\infty} a_{\mu, v} \varepsilon^{2}+o\left(\varepsilon^{2}\right)<\frac{\alpha}{2(N+\alpha)} c_{\infty}^{-\frac{N}{\alpha}},
$$

where $C_{16}$ is independent of $\varepsilon$.

If $N=3$, we have $\frac{N(N+\alpha)}{N+2 \alpha}>2$ and $\frac{N^{2}}{2 N-\alpha}>2$ if $\frac{3}{2}<\alpha<3$. By (3.13), for all $v \in E^{-}$and $t \in\left[t_{\star}, t^{\star}\right]$, as $\varepsilon \rightarrow 0$,

$$
\begin{aligned}
J\left(t v_{\varepsilon}+v\right) \leq & \frac{1}{2} t^{2}-\frac{N}{2(N+\alpha)} J_{1}+C_{11} t^{\frac{2(N+\alpha)}{N+2 \alpha}} \varepsilon^{\frac{N(N+\alpha)}{N+2 \alpha}} \\
& +\frac{N}{2(N+\alpha)}\left(C_{15} t^{\star} \frac{2 \alpha^{2}}{N(2 N-\alpha)} \varepsilon^{\frac{N^{2}}{2 N-\alpha}}+C_{13} \varepsilon^{N+\alpha}\right) t^{\star \frac{2(N+\alpha)}{N}} \\
= & \frac{1}{2} t^{2}-\frac{N}{2(N+\alpha)} c_{\infty} t^{\frac{2(N+\alpha)}{N}}+\frac{1}{2} t_{\star}^{\frac{2(N+\alpha)}{N}} c_{\infty} a_{\mu, v} \varepsilon^{2}+o\left(\varepsilon^{2}\right) .
\end{aligned}
$$


Similarly as above, $\sup _{w \in \hat{E}\left(v_{\varepsilon}\right)} J(w)<\frac{\alpha}{2(N+\alpha)} c_{\infty}^{-\frac{N}{\alpha}}$ for $\varepsilon>0$ small.

Case 2. $0<\beta<2, v_{\beta}>0$.

Similarly as above, if $N \geq 4$, for all $v \in E^{-}$, as $\varepsilon \rightarrow 0$, there holds that

$$
\begin{aligned}
J\left(t v_{\varepsilon}+v\right) \leq \frac{1}{2} t^{2}-\frac{N}{2(N+\alpha)} t^{\frac{2(N+\alpha)}{N}} & \left(c_{\infty}+b_{\beta, \alpha} v_{\beta} \varepsilon^{\beta}-C_{12} \varepsilon^{\frac{N(N+\alpha)}{2 N+\alpha}}\right. \\
& \left.+o\left(\varepsilon^{\beta}\right)+o\left(\varepsilon^{\frac{N(N+\alpha)}{2 N+\alpha}}\right)\right), t \in\left[t_{*}, t^{\star}\right] .
\end{aligned}
$$

Recalling that $\frac{N(N+\alpha)}{2 N+\alpha}>2$ when $N \geq 4$, which implies that

$$
J\left(t v_{\varepsilon}+v\right) \leq \frac{1}{2} t^{2}-\frac{N}{2(N+\alpha)} t^{\frac{2(N+\alpha)}{N}}\left(c_{\infty}+b_{\beta, \alpha} v_{\beta} \varepsilon^{\beta}+o\left(\varepsilon^{\beta}\right)\right), t \in\left[t_{*}, t^{*}\right] .
$$

Similarly as above, we get that $\sup _{w \in \hat{E}\left(v_{\varepsilon}\right)} J(w)<\frac{\alpha}{2(N+\alpha)} c_{\infty}^{-\frac{N}{\alpha}}$, for $\varepsilon>0$ small.

If $N=3$, similarly as above, for all $v \in E^{-}$and $t \in\left[t_{\star}, t^{\star}\right]$, as $\varepsilon \rightarrow 0$,

$$
\begin{aligned}
J\left(t v_{\varepsilon}+v\right) \leq & \frac{1}{2} t^{2}-\frac{N}{2(N+\alpha)} t^{\frac{2(N+\alpha)}{N}}\left(c_{\infty}+b_{\beta, \alpha} v_{\beta} \varepsilon^{\beta}+o\left(\varepsilon^{\beta}\right)\right) \\
& +\frac{N}{2(N+\alpha)} C_{15} t^{\star \frac{4 N+2 \alpha}{2 N-\alpha}} \varepsilon^{\frac{N^{2}}{2 N-\alpha}} \\
= & \frac{1}{2} t^{2}-\frac{N}{2(N+\alpha)} c_{\infty} t^{\frac{2(N+\alpha)}{N}}-\frac{N}{2(N+\alpha)} t_{\star}^{\frac{2(N+\alpha)}{N}} b_{\beta, \alpha} v_{\beta} \varepsilon^{\beta} \\
& +\frac{N}{2(N+\alpha)} C_{15} t^{\star \frac{4 N+2 \alpha}{2 N-\alpha}} \varepsilon^{\frac{N^{2}}{2 N-\alpha}}+o\left(\varepsilon^{\beta}\right) .
\end{aligned}
$$

It follows that if $\beta<\frac{N^{2}}{2 N-\alpha}$, for all $v \in E^{-}$and $t \in\left[t_{\star}, t^{\star}\right]$, as $\varepsilon \rightarrow 0$,

$$
J\left(t v_{\varepsilon}+v\right) \leq \frac{1}{2} t^{2}-\frac{N}{2(N+\alpha)} c_{\infty} t^{\frac{2(N+\alpha)}{N}}-\frac{N}{2(N+\alpha)} t_{\star}^{\frac{2(N+\alpha)}{N}} b_{\beta, \alpha} v_{\beta} \varepsilon^{\beta}+o\left(\varepsilon^{\beta}\right) .
$$

Similarly as above, $\sup _{w \in \hat{E}\left(v_{\varepsilon}\right)} J(w)<\frac{\alpha}{2(N+\alpha)} c_{\infty}^{-\frac{N}{\alpha}}$ for $\varepsilon>0$ small. The proof is complete.

\subsection{Palais-Smale condition.}

Similarly to [7], we have the following compactness result, which plays a crucial role in finding nontrivial solutions of (1.8).

Lemma 3.2. The functional $J$ satisfies the Palais-Smale condition in $(-\infty, c)$ if $c<\frac{\alpha}{2(N+\alpha)} c_{\infty}^{-\frac{N}{\alpha}}$. Namely, if $\left\{u_{m}\right\}_{m \in \mathbb{N}} \subset H^{1}\left(\mathbb{R}^{N}\right)$ satisfies

$$
J\left(u_{m}\right) \rightarrow c, J^{\prime}\left(u_{m}\right) \rightarrow 0 \text { in } H^{-1}\left(\mathbb{R}^{N}\right) \text {, as } m \rightarrow \infty
$$

then up to a subsequence, there exists $u \in H^{1}\left(\mathbb{R}^{N}\right)$ such that $u_{m} \rightarrow u$ strongly in $H^{1}\left(\mathbb{R}^{N}\right)$, as $m \rightarrow \infty$.

Proof of Lemma 3.2. The proof is similar to [7, Lemma 3.7]. For the sake of completeness, we give just a sketch. Let $\left\{u_{m}\right\}_{m \in \mathbb{N}} \subset H^{1}\left(\mathbb{R}^{N}\right)$ be a (P-S) $c$ sequence, namely

$$
J\left(u_{m}\right) \rightarrow c<\frac{\alpha}{2(N+\alpha)} c_{\infty}^{-\frac{N}{\alpha}}, J^{\prime}\left(u_{m}\right) \rightarrow 0 \text { in } H^{-1}\left(\mathbb{R}^{N}\right) \text {, as } m \rightarrow \infty .
$$

Then by a similar fashion, we know that the sequence $\left\{u_{m}\right\}_{m \in \mathbb{N}}$ is bounded in $H^{1}\left(\mathbb{R}^{N}\right)$ and up to a subsequence, there exists $u \in H^{1}\left(\mathbb{R}^{N}\right)$ such that $u_{m} \rightarrow u$ weakly in $H^{1}\left(\mathbb{R}^{N}\right)$ and almost everywhere in $\mathbb{R}^{N}$, as $m \rightarrow \infty$. Let $v_{m}=u_{m}-u$, then by the weak convergence and a Brezis-Lieb type lemma [19, Lemma 2.4],

$$
\left\{\begin{array}{l}
c+o_{m}(1)=J(u)+\frac{1}{2}\left\|v_{m}\right\|^{2}-\frac{N}{2(N+\alpha)} G_{\infty}\left(v_{m}\right), \\
o_{m}(1)=\left\langle J^{\prime}(u), u\right\rangle+\left\|v_{m}\right\|^{2}-G_{\infty}\left(v_{m}\right) .
\end{array}\right.
$$


Since $J^{\prime}(u)=0$ in $H^{-1}\left(\mathbb{R}^{N}\right)$,

$$
\begin{aligned}
J(u) & =J(u)-\frac{N}{2(N+\alpha)}\left\langle J^{\prime}(u), u\right\rangle \\
& =\frac{\alpha}{2(N+\alpha)} \int_{\mathbb{R}^{N}}|\nabla u|^{2}+V_{\mu, v}|u|^{2} \geq 0 .
\end{aligned}
$$

Suppose $G_{\infty}\left(v_{m}\right) \rightarrow l \geq 0$, as $m \rightarrow \infty$, then by (3.14) $\lim _{m \rightarrow \infty}\left\|v_{m}\right\|^{2}=l$. If $l>0$, then

$$
l+o_{m}(1)=G_{\infty}\left(v_{m}\right) \leq c_{\infty}\left\|v_{m}\right\|_{2}^{\frac{2(N+\alpha)}{N}} \leq c_{\infty}\left(l+o_{m}(1)\right)^{\frac{N+\alpha}{N}},
$$

which implies $l \geq c_{\infty}^{-\frac{N}{\alpha}}$. Then by (3.14),

$$
c \geq \frac{\alpha}{2(N+\alpha)} c_{\infty}^{-\frac{N}{\alpha}},
$$

which is a contradiction. Therefore $l=0$ and the proof is complete.

Proof of Theorem 1.3. Now, we are in position to prove Theorem 1.3. By virtue of [3, Theorem 2.4] due to P. Bartolo, V. Benci and D. Fortunato, similarly as that in [7], (1.8) admits at least one nontrivial solution $u \in H^{1}\left(\mathbb{R}^{N}\right)$ with $J(u)<\frac{\alpha}{2(N+\alpha)} c_{\infty}^{-\frac{N}{\alpha}}$. Let

$$
K:=\left\{u \in H^{1}\left(\mathbb{R}^{N}\right) \backslash\{0\}: J^{\prime}(u)=0 \text { in } H^{-1}\left(\mathbb{R}^{N}\right)\right\},
$$

then $K \neq \emptyset$ and

$$
m:=\inf _{u \in K} J(u)<\frac{\alpha}{2(N+\alpha)} c_{\infty}^{-\frac{N}{\alpha}} .
$$

Similarly to [7], $m>0$ and there exists $u_{0} \in H^{1}\left(\mathbb{R}^{N}\right)$ such that $u_{0} \in K$ and $J\left(u_{0}\right)=m$. The proof of Theorem 1.3 is complete.

Acknowledgements: Zhisu Liu is supported by the NSFC (11626127), Natural Science Foundation of Hunan Province (2019JJ50490; 2020JJ3029), Scientific Research Foundation of Hunan Province Education Department (Grant No. 18C0455), and the Fundamental Research Funds for the Central Universities, China University of Geosciences (Wuhan, Grant number: CUGST2). J. Zhang was partially supported by NSFC (11871123) and Team Building Project for Graduate Tutors in Chongqing (JDDSTD201802).

Conflict of interest statement: Authors state no conflict of interest.

\section{References}

[1] C. O. Alves, D. Cassani, C. Tarsi and M. Yang, Existence and concentration of ground state solutions for a critical nonlocal Schrödinger equation in $\mathbb{R}^{2}$, J. Differential Equations, 261(2016), 1933-1972.

[2] C. O. Alves and M. Yang, Existence of solutions for a nonlocal variational problem in R2 with exponential critical growth, J. Convex Anal. 24 (2017), 1197-1215.

[3] P. Bartolo, V. Benci and D. Fortunato, Abstract critical point theorems and applications to some nonlinear problems with "strong" resonance at infinity, Nonlinear Analysis: Theory, Methods and Applications, 7 (1983), 981-1012.

[4] J. Byeon and L. Jeanjean, Standing waves for nonlinear Schödinger equations with a general nonlinearity. Arch. Ration. Mech. Analysis, 185(2007), 185-200.

[5] D. Bonheure, S. Cingolani and J. Van Schaftingen, The logarithmic Choquard equation: sharp asymptotics and nondegeneracy of the groundstate, J. Funct. Anal. 272(2017), 5255-5281.

[6] D. Cassani and J.J. Zhang, Choquard-type equations with Hardy-Littlewood-Sobolev upper-critical growth, Advances in Nonlinear Analysis, 8 (2019), 1184-1212.

[7] D. Cassani, J. Van Schaftingen and J. J. Zhang, Groundstates for Choquard type equations with Hardy-Littlewood-Sobolev lower critical exponent, Proceedings of the Royal Society of Edinburgh, Section A Mathematics, 150(2020), 1377-1400.

[8] S. Chen, X. Tang and J. Wei, Nehari-type ground state solutions for a Choquard equation with doubly critical exponents, Adv. Nonlinear Anal. 10 (2021), 152-171. 
[9] F. Gao and M. Yang, The Brezis-Nirenberg type critical problem for the nonlinear Choquard equation, Sci. China Math. 61 (2018), 1219-1242.

[10] E. H. Lieb and M. Loss, Analysis, 2nd edn, Graduate Studies in Mathematics, vol. 14. American Mathematical Society, Providence, 2001.

[11] E.H. Lieb and B. Simon, The Hartree-Fock theory for Coulomb systems, Comm. Math. Phys., 53(1977), 185-194.

[12] E.H. Lieb, Existence and uniqueness of the minimizing solution of Choquard's nonlinear equation, Stud. Appl. Math., 57 (1976/77), 93-105.

[13] P. L. Lions, The concentration-compactness principle in the calculus of variations. The locally compact case I. II, Annales Inst. H. Poincaré Analyse Non Linéaire, 1 (1984), 109-145, 223-283.

[14] P.L. Lions, The Choquard equation and related questions, Nonlinear Anal. TMA, 4 (1980), 1063-1073.

[15] P.L. Lions, Compactness and topological methods for some nonlinear variational problems of mathematical physics, Nonlinear problems: present and future 17-34, 1982.

[16] S. Liang, P. Pucci and B. Zhang, Multiple solutions for critical Choquard-Kirchhoff type equations, Adv. Nonlinear Anal. 10 (2021), 400-419.

[17] X. He and Vicenţiu D. Rădulescu, Small linear perturbations of fractional Choquard equations with critical exponent, $J$. Differential Equations, 282(2021), 481-540.

[18] C. Mercuri, V. Moroz and J. Van Schaftingen, Groundstates and radial solutions to nonlinear Schrödinger-Poisson-Slater equations at the critical frequency, Calc. Var. Partial Differential Equations, 55 (2016), no. 146.

[19] V. Moroz and J. Van Schaftingen, Groundstates of nonlinear Choquard equations: existence, qualitative properties and decay asymptotics, J. Funct. Anal., 265 (2013), 153-184.

[20] V. Moroz and J. Van Schaftingen, Groundstates of nonlinear Choquard equations: Hardy-Littlewood-Sobolev critical exponent, Comm. Contem. Math., 17 (2015), 1550005 (12 pages).

[21] V. Moroz and J. Van Schaftingen, A guide to the Choquard equation, J. Fixed Point Theory Appl., 19 (2017), 773-813.

[22] V. Moroz and J. Van Schaftingen, Existence of groundstates for a class of nonlinear Choquard equations, Trans. Am. Math. Soc., 367(9) (2015), 6557-6579.

[23] S. Pekar, Untersuchung über die Elektronentheorie der Kristalle, Akademie Verlag, Berlin (1954).

[24] J. Seok, Nonlinear Choquard equations: doubly critical case, Appl. Math. Lett., 76(2018), 148-156.

[25] J. Van Schaftingen and J. Xia, Groundstates for a local nonlinear perturbation of the Choquard equations with lower critical exponent, J. Math. Anal. Appl. 464 (2018), 1184-1202.

[26] Z. Yang and F. Zhao, Multiplicity and concentration behaviour of solutions for a fractional Choquard equation with critical growth, Adv. Nonlinear Anal. 10 (2021), 732-774. 